The Journal of Nonlinear $\mathbf{S}$ ciences and Applications http://www.tjnsa.com

\title{
WHEN IS A QUASI-P-PROJECTIVE MODULE DISCRETE?
}

\author{
Y. TALEBI ${ }^{1 *}$ AND I. KHALILI GORJI ${ }^{2}$
}

\begin{abstract}
It is well-known that every quasi-projective module has $D_{2}$-condition. In this note it is shown that for a quasi-p-projective module $M$ which is selfgenerator, duo, then $M$ is discrete.
\end{abstract}

\section{INTRODUCTION AND PRELIMINARIES}

Throughout, $R$ is an associative ring with identity and right $R$-modules are unitary. Let $M$ be a right $R$-module. A module $N$ is called $M$-generated if there is an epimorphism $M^{(I)} \longrightarrow N$ for some index set $I$. In particular, $N$ is called $M$-cyclic if it is isomorphism to $M / L$ for submodule $L \subseteq M$. Following [3] a module $M$ is called self-generate if it generates all its submodules. For standard notation and terminologies, we refer to [4], 3].

Let $M$ be a right $R$-module. A right $R$-module $N$ is called $M$-p-projective if every homomorphism from $N$ to an $M$-cyclic submodule of $M$ can be lifted to an $R$-homomorphism from $N$ to $M$. A right $R$-module $M$ is called quasi-p-projective , if it is $M$-p-projective. A submodule $A$ of $M$ is said to be a small submodule of $M$ (denoted by $A \ll M$ ) if for any $B \subseteq M, A+B=M$ implies $B=M$. A module $M$ is called hollow if every its submodule is small.

In [2], S.Chotchaisthit showed that a quasi-p-injective module $M$ is continouse, if $M$ is duo and semiprefect. Here we study, when a quasi-p-projective module is discrete.

Consider the following conditions for a module $M$ which have studied in [3] :

$D_{1}$ : For every submodules $N$ of $M$ there exist submodules $K, L$ of $M$ such that $M=K \oplus L$ and $K \leq N$ and $N \cap L \ll L$.

Date: Received: 27 September 2008.

* Corresponding author.

2000 Mathematics Subject Classification. Primary 16D40; Secondary 16D60, 16D90.

Key words and phrases. Supplemented Module, H-Supplemented Module, Lifting Module. 
$D_{2}$ : If $N$ is a submodule of $M$ such that $M / N$ is isomorphism to a direct summand of $M$, then $N$ is a direct summand of $M$.

$D_{3}$ : For every direct summands $K, L$ of $M$ with $M=K+L, K \cap L$ is a direct summand of $M$.

If the module $M$ satisfies $D_{1}$ and $D_{2}$ then it is called a discrete module.

It is clear that if $M$ is hollow, then it has $D_{1}$ and $D_{2}$ conditions, since hollow module is indecomposition.

\section{MAin RESUlts}

Recall that a submodule $N$ of $M$ is called a fully invariant submodule if $s(N) \subseteq N$, for any endomorphism $s$ of $M$. A right $R$-module is called a $d u o$ module if every submudole is fully invariant. A ring $R$ is right duo if every right ideal is two sided. The proof of the following Lemma is routine.

Lemma 2.1. Let $M$ be a duo right $R$-module and $A$ its direct summand. Then:

(1) A is itself a duo module;

(2) If $M$ is a self-generator, then $A$ is also a self-generator.

Proof. (1) Let $f \in \operatorname{End}(A), \pi: M \rightarrow A, i: A \rightarrow M$ be the projection and inclusion maps. Then $g=i f \pi \in \operatorname{End}(M)$. It follows that for any submodule $X$ of $A, f(X)=g(X) \subset X$, proving our Lemma.

(2) Let $M=A \oplus B$. Then $f(M)=f(A)+f(B)$ for any $f \in \operatorname{End}(M)$. Let $X$ be a submodule of $A$. Since $M$ is a self-generator, we can write $X=\sum_{f \in I} f(M)=$ $\sum_{f \in I}(f(A)+f(B))$, for some subset $I$ of $\operatorname{End}(M)$. Since $f(B) \subset B$, it follows that $f(B)=0$ for all $f \in I$. Hence $X=\sum_{f \in I} f(A)$. Moreover, $f$ can be considered as an endomorphism of $A$, since $f(A) \subset A$. This shows that $A$ is a self-generator.

Lemma 2.2. Let $M$ be a quasi-p-projective. If $S=\operatorname{End}\left(M_{R}\right)$ is local, then for any non-traivial fully invariant $M$-cyclic submodules $A$ and $B$ of $M, A+B \neq M$.

Proof. let $0 \neq s(M)=A$ and $0 \neq t(M)=B, s, t \in S$ and $A+B=M$. Difine the map $f: M=(s+t)(M) \rightarrow M /(A \cap B)$ such that $f(s+t)(m)=s(m)+(A \cap B)$. For any $m, m^{\prime} \in M,(s+t)(m)=(s+t)\left(m^{\prime}\right)$ implies $s\left(m-m^{\prime}\right)=t\left(m^{\prime}-m\right) \in A \cap B$. So $s(m)+(A \cap B)=s\left(m^{\prime}\right)+(A \cap B)$. Clearly $f$ is an $R$-homomorphism. By quasi-pprojective, there exist $g \in S$ such that $\pi \circ g=f$ and $\pi: M \rightarrow M /(A \cap B)$ is natural epimorphism . It follows $\pi \circ g(s+t))(m)=\pi(s(m))$. Then $((1-g) \circ s-g \circ t)(M) \subseteq$ $(A \cap B)$. Since $S$ is local, $g$ or $1-g$ is invertible. If $1-g$ be invertible we have $\left(s-(1-g)^{-1} \circ g \circ t\right)(M) \subseteq(A \cap B) . A \subseteq\left(s-(1-g)^{-1} \circ g \circ t\right)(M) \subseteq(1-g)^{-1}(A \cap B) \subseteq$ $(A \cap B)$. Then $A \subseteq(A \cap B)$, that is contradiction. If $g$ be invertible we have $B \subseteq\left(g^{-1} \circ(1-g) \circ s-t\right) \subseteq g^{-1}(A \cap B) \subseteq(A \cap B)$. Then $B \subseteq(A \cap B)$, that is contradiction.

Corollary 2.3. If $M$ is qasi-p-projective duo module which is a self-generator with local endomorphism ring, then $M$ is hollow, hence it is discrete.

Proof. It is clear by Lemma 2.2

Lemma 2.4. Let $M=\oplus_{i \in I} B_{i}$ be duo module. Then for any submodule $A$ of $M$ we have $A=\oplus_{i \in I}\left(A \cap B_{i}\right)$. 
Proof. See [1].

Corollary 2.5. Let $M$ be a duo module. If $A$ and $B$ are direct summands of $M$, then so $A \cap B$.

Proof. Let $M=A \oplus A_{1}=B \oplus B_{1}$, then by lemma $2.4 B=B \cap\left(A \oplus A_{1}=\right.$ $(A \cap B) \oplus\left(B \cap A_{1}\right)$. hence $M=(A \cap B) \oplus\left(B \cap A_{1}\right) \oplus B_{1}$. So $A \cap B$ is a direct summand of $M$.

Theorem 2.6. Let $M=\oplus_{i \in I} M_{i}$ be qusi-p-projective module where each $M_{i}$ is hollow. If $M$ is duo module, $\operatorname{Rad}(M) \ll M$ then $M$ is discrete.

Proof. By Lemma 2.4 every submodule $A$ of $M$ can be written in the form $A=$ $\oplus_{j \in J}\left(A \cap M_{j}\right)$ where $J \subseteq I$ and $A \cap M_{j} \neq 0$. Since $A \cap M_{j}$ is small in $M_{j}$ we see that $A$ is small in $M$. Thus we have proved.

Theorem 2.7. Suppose that $M$ is semisimple quasi-p-projective duo module and $\operatorname{Rad}(M) \ll M$. If $M$ is self-generator, then $M$ is discrete.

Proof. We have $M=\oplus_{i \in I} M_{i}$ such that $M_{i}$ is simple, then $\operatorname{End}\left(M_{i}\right)$ is local. By Lemma 2.1 each $M_{i}$ is duo and self-generator. Since any direct summand of a quasi-p-projective is again quasi-p-projective, it follows from Corollary 2.3 that each $M_{i}$ is discrete. From Theorem 2.6 that $M$ is discrete, proving our Theorem.

Acknowledgements. This research partially is supported by the "research center in Algebraic Hyperstructure and Fuzzy Mathematics University of Mazandaran, Babolsar, Iran".

\section{REFERENCES}

1. G. F. Birkenmeier, B. J. Muller and S. T. Rizvi, Modules in which Every Fully Invariant submodule is Essential in a Fully Invariant Direct Summand, Comm. Algebra, 30 (2002) 1833-1852. 2

2. S. Chotchaisthit, When is a Quasi-p-injective Module Continuous?, Southest Asian Bulletin of Mathematics 26 (2002) 391-394. 1

3. S. M. Mohamed, and B. J. Muller, Continuous and Discrete Modules, London Math, Soc, Lecture Notes Series 147, Cambridge, University Press, (1990). 1

4. R. Wisbauer, Foundations of Module and Ring Theory, Gordon and Breach, Philadelphia,(1991). 1

${ }^{1}$ Department of Mathematics, University of Mazandaran, Babolsar, Iran.

E-mail address: talebi@umz.ac.ir 